\title{
Yamabe Constants and the Perturbed Seiberg-Witten Equations
}

\author{
Claude LeBrun ${ }^{1}$
}

\begin{abstract}
Among all conformal classes of Riemannian metrics on $\mathbf{C P}_{2}$, that of the Fubini-Study metric is shown to have the largest Yamabe constant. The proof, which involves perturbations of the SeibergWitten equations, also yields new total scalar curvature bounds for other 4-manifolds.
\end{abstract}

\section{Introduction.}

One may define an interesting and natural diffeomorphism invariant of a compact smooth $n$-manifold $M$ as a minimax of the total scalar curvature over all unit volume Riemannian metrics on $M$. To be precise, for each Riemannian metric $g$ on $M$, one may first define an invariant of the corresponding conformal class $[g]=\left\{v g \mid v: M \rightarrow \mathbf{R}^{+}\right\}$by setting

$$
Y_{[g]}=\inf _{g \in[g]} \frac{\int_{M} s_{g} d \mu_{g}}{\left(\int_{M} d \mu_{g}\right)^{\frac{n-2}{n}}}
$$

where $s_{g}$ and $d \mu_{g}$ respectively denote the scalar curvature and volume $n$ form of $g$. The number $Y_{[g]}$ is called the Yamabe constant of the conformal class of metrics, and a remarkable theorem $[1,14,19]$ of Yamabe, Trudinger, Aubin, and Schoen states that any conformal class $[g]$ contains a constantscalar-curvature metric which achieves this infimum. We may now define an invariant

$$
Y(M)=\sup _{[g]} Y_{[g]}=\sup _{[g]} \inf _{g \in[g]} \frac{\int_{M} s_{g} d \mu_{g}}{\left(\int_{M} d \mu_{g}\right)^{\frac{n-2}{n}}}
$$

which we will call the Yamabe invariant of $M$.

For 2-manifolds, this invariant is easy to compute; the Gauss-Bonnet theorem tells us that the Yamabe invariant of a compact surface of genus $p$ is

\footnotetext{
${ }^{1}$ Supported in part by NSF grant DMS-9505744.
} 
just $8 \pi(1-p)$. But relatively few precise computations of Yamabe invariants have proved tractable for manifolds of higher dimension. One important general fact, due to Aubin, is that for any compact $n$-manifold one has $Y(M) \leq Y\left(S^{n}\right)=n(n-1) V_{n}^{2 / n}$, where $V_{n}$ is the volume of the unit $n$-sphere. It has also been known for some time $[8,20]$ that the $n$-torus and certain other manifolds with Ricci-flat metrics have $Y=0$, and the very first result in this direction [16] crucially depended on the introduction of the Dirac operator. It was nonetheless a revolutionary development when Witten [25] observed that a non-linear Dirac system called the Seiberg-Witten equations can allow one to distinguish different smooth structures on a topological 4-manifold by virtue of the fact that the corresponding Yamabe invariants are distinct. Pushing this insight to its logical conclusion resulits in a precise computation [13] of the Yamabe invariant for a large class of 4-manifolds with $Y(M)<0$.

The usual Seiberg-Witten invariant vanishes, however, when $Y(M)>$ 0 , so it might seem hopeless to try to use such techniques to show that certain 4-manifolds have $0<Y(M)<Y\left(S^{4}\right)=8 \sqrt{6} \pi$. For 4-manifolds with $b_{+}=1$, however, it turns out that a perturbed version of the equations $[23,11,15]$ gives rise to an invariant which is often non-trivisl even in the presence of metrics of positive scalar curvature. Extracting; information about the scalar curvature becomes more complicated, however, since the perturbation dominates the metric terms in the corresponding Weitzenböck formula. Nonetheless, if the perturbed Seiberg-Witten equations on $\left(M^{4}, g\right)$ have a solution when the perturbation term is any large multiple of the self-dual harmonic form $\omega$, then one obtains ( 22 the basic estimate

$$
\int s \frac{|\omega|}{\sqrt{2}} d \mu \leq 4 \pi c_{1} \cdot[\omega],
$$

with equality if $g$ is Kähler. The presence of $\omega$ in this estimate exactly compensates for the fact that the Yamabe functional is not bounded above. One eventually estimates the Yamabe constants $Y_{[g]}$ by $(\S 4)$ representing each conformal class by metrics for which the size of $\omega$ is nearly constant. The main application is the following:

Theorem A. The Yamabe invariant of the complex projective plane is given by

$$
Y\left(\mathbf{C P}_{2}\right)=12 \sqrt{2} \pi .
$$

Moreover, a conformal class [g] on $\mathbf{C P}_{2}$ satisfies $Y_{[g]}=Y\left(\mathbf{C P}_{2}\right)$ iff there is a diffeomorphism $\Phi: \mathbf{C P}_{2} \rightarrow \mathbf{C P}_{2}$ such that $\Phi^{*}[g]$ is the conformal class of the Fubini-Study metric. 
The basic estimate, however, has other interesting applications, notably to the theory of almost-Kähler 4-manifolds.

\section{The Basic Estimate.}

Let $M^{4}$ be a compact oriented 4-manifold with $b^{+}=1$. The open cone

$$
\left\{[\omega] \in H^{2}(M ; \mathbf{R}) \mid[\omega] \cdot[\omega]>0\right\}
$$

then consists of two connected components, called nappes. Given such a nappe $\mathcal{C}^{+}$, and a Riemannian metric $g$ on $M$, let $\omega$ be a $g$-harmonic 2form such that $[\omega] \in \mathcal{C}^{+}$; of course, such a form always exists, and it is uniquely determined once we also specify the positive real number $[\omega]^{2}$. If $c$ is the $\operatorname{spin}^{c}$ structure induced by some almost-complex structure $J$ on $M$, then, relative to any metric $g$, we can define the (perturbed) Seiberg-Witten invariant $p_{c}\left(M, \mathcal{C}^{+}\right)$as the number of solutions, modulo gauge and counted with orientations, of the perturbed Seiberg-Witten equations $[10,25]$

$$
\begin{aligned}
D_{A} \Phi & =0 \\
i F_{A}^{+}+\sigma(\Phi) & =\varepsilon
\end{aligned}
$$

where $\varepsilon$ is a generic self-dual 2-form with $\int_{M} \varepsilon \wedge \omega>2 \pi c_{1} \cdot[\omega]$. Here $\omega$ is a $g$-self-dual 2-form with $[\omega] \in \mathcal{C}^{+}$. More generally, if $c$ is a spin ${ }^{c}$ structure for which $k=\left[c_{1}^{2}-(2 \chi+3 \tau)(M)\right] / 4$ is non-negative and even, one can define $[15,22]$ the perturbed Seiberg-Witten invariant $p_{c}\left(M, \mathcal{C}^{+}\right)$to be $\int_{X} \alpha^{k / 2}$, where $X$ is the moduli space of solutions of (2.1-2.2) for a generic $\varepsilon$ with $\int \varepsilon \wedge \omega>2 \pi c_{1} \cdot[\omega]$, and $\alpha \in H^{2}(X)$ is the first Chern class of the based moduli space, considered as an $S^{1}$-bundle over $X$. For our purposes, the key point is simply that when this invariant is non-zero, the equations

$$
\begin{aligned}
D_{A} \Phi & =0 \\
-i F_{A}^{+} & =\sigma(\Phi)-t \omega,
\end{aligned}
$$

have a solution with $\Phi \not \equiv 0$ for any $t \gg 0$.

Theorem 1. Let $M^{4}$ be a smooth compact oriented 4-manifold with $b^{+}=1$ for which there is a nappe $\mathcal{C}^{+}$and a spin ${ }^{c}$ structure $c$ such that $p_{c}\left(M, \mathcal{C}^{+}\right) \neq$ 0 . Let $g$ be any Riemannian metric on $M$, and let $\omega$ be a g-self-dual harmonic 2-form with $[\omega] \in \mathcal{C}^{+} \subset H^{2}(M, \mathbf{R})$. Then the scalar curvature $s$ of $g$ satisfies

$$
\int s \frac{|\omega|}{\sqrt{2}} d \mu \leq 4 \pi c_{1} \cdot[\omega]
$$


Here $d \mu$ and $|\cdot|$ are respectively the volume form and point-wise norm determined by the metric $g$, while $c_{1}=c_{1}\left(V_{+}\right)$is the first Chern class of the spin $^{c}$ structure.

Proof. The Dirac equation $D_{A} \Phi=0$ implies the Weitzenböck formula

$$
0=\langle\Phi, \Delta \Phi\rangle+\frac{s}{4}|\Phi|^{2}+2\left\langle-i F_{A}^{+}, \sigma(\Phi)\right\rangle,
$$

where the natural real-quadratic map $\sigma: V_{+} \rightarrow \wedge^{+}$satisfies $|\sigma(\Phi)|=$ $2^{-3 / 2}|\Phi|^{2}$. For a solution of the perturbed Seiberg-Witten equations (2.32.4), we also have $-i F_{A}^{+}=\sigma(\Phi)-t \omega$, so it follows that

$$
\begin{aligned}
0 & =\int_{M}\left[4\langle\Phi, \Delta \Phi\rangle+s|\Phi|^{2}+8\langle\sigma(\Phi)-t \omega, \sigma(\Phi)\rangle\right] d \mu \\
& =\int_{M}\left[\left|2 \nabla_{A} \Phi\right|^{2}+s|\Phi|^{2}+|\Phi|^{4}-8 t\langle\omega, \sigma(\Phi)\rangle\right] d \mu \\
& \geq \int_{M}\left[s|\Phi|^{2}+|\Phi|^{4}-8 t|\omega||\sigma(\Phi)|\right] d \mu \\
& =\int_{M}\left[s|\Phi|^{2}+|\Phi|^{4}-2 \sqrt{2} t|\omega||\Phi|^{2}\right] d \mu
\end{aligned}
$$

Hence

and so the Cauchy-Schwarz inequality yields

$$
\int_{M}(2 \sqrt{2} t|\omega|-s)|\Phi|^{2} d \mu \geq \int_{M}|\Phi|^{4} d \mu
$$

$$
\begin{aligned}
8 t^{2}[\omega]^{2}-4 \sqrt{2} t \int_{M} s|\omega| d \mu & +\int_{M} s^{2} d \mu=\int(2 \sqrt{2} t|\omega|-s)^{2} d \mu \\
& \geq \frac{\left[\int|\Phi|^{4} d \mu\right]^{2}}{\int|\Phi|^{4} d \mu} \\
& =\int|\Phi|^{4} d \mu \\
& =8 \int|\sigma(\Phi)|^{2} d \mu \\
& =8 \int\left|-i F_{A}^{+}+t \omega\right|^{2} d \mu \\
& =8 \int\left(t^{2}|\omega|^{2}-2 t\left\langle i F_{A}^{+}, \omega\right\rangle+\left|i F_{A}^{+}\right|^{2}\right) d \mu \\
& =8\left(t^{2}[\omega]^{2}-2 t\left(2 \pi c_{1}\right) \cdot[\omega]+\int\left|i F_{A}^{+}\right|^{2} d \mu\right) \\
& \geq 8 t^{2}[\omega]^{2}-32 \pi t c_{1} \cdot[\omega]+32 \pi^{2} c_{1}^{2} .
\end{aligned}
$$


for all $t \gg 0$. Hence

$$
32 \pi t c_{1} \cdot[\omega]+\int_{M} s^{2} d \mu \geq 4 \sqrt{2} t \int_{M} s|\omega| d \mu+32 \pi^{2} c_{1}^{2}
$$

for all $t \gg 0$. Dividing by $8 t$ and taking the limit as $t \rightarrow+\infty$, we therefore have $4 \pi c_{1} \cdot[\omega] \geq \int_{M} s \frac{|\omega|}{\sqrt{2}} d \mu$, as claimed.

Theorem 2. Equality holds in Theorem 2 iff $g$ is Kähler with respect to some c-compatible complex structure $J$ and $\omega$ is a constant positive multiple of the Kähler form of $(M, g, J)$.

Proof. The Weitzenböck formula for (2.3-2.4) tells us that

$$
\begin{aligned}
0 & =2 \Delta|\Phi|^{2}+|2 \nabla \Phi|^{2}+s|\Phi|^{2}+|\Phi|^{4}-8 t\langle\omega, \sigma(\Phi)\rangle \\
& \geq 2 \Delta|\Phi|^{2}+s|\Phi|^{2}+|\Phi|^{4}-2 \sqrt{2} t|\omega||\Phi|^{2}
\end{aligned}
$$

so that

$$
|\Phi|^{2} \leq 2 \sqrt{2} t|\omega|-s
$$

at the maximum of $|\Phi|$. The $C^{0}$ norm of the twisted spinor field $\Psi_{t}=\Phi / \sqrt{t}$ is therefore uniformly bounded as $t \rightarrow \infty$ :

$$
\left|\Psi_{t}\right|^{2} \leq 2 \sqrt{2} \max |\omega|-\min (\min s, 0) \forall t>1 .
$$

Now the proof of Theorem 2 contains the inequality

$$
8 t^{2}[\omega]^{2}-4 \sqrt{2} t \int_{M} s|\omega| d \mu+\int_{M} s^{2} d \mu \geq 8\left(t^{2}[\omega]^{2}-2 t\left(2 \pi c_{1}\right) \cdot[\omega]+\int_{M}\left|i F_{A_{t}}^{+}\right|^{2} d \mu\right)
$$

and it therefore follows that

$$
\int_{M} s \frac{|\omega|}{\sqrt{2}} d \mu=4 \pi c_{1} \cdot[\omega] \Longrightarrow\left\|i F_{A}^{+}\right\|_{L^{2}}^{2} \leq \frac{1}{8} \int_{M} s^{2} d \mu
$$

Since equation (2.4) stipulates that

$$
\sigma\left(\Psi_{t}\right)-\omega=\frac{\sigma(\Phi)-t \omega}{t}=-\frac{i F_{A}^{+}}{t}
$$

equality in Theorem 2 implies that $\sigma\left(\Psi_{t}\right) \rightarrow \omega$ in $L^{2}$.

On the other hand, the proof of Theorem 2 neglected the $|\nabla \Phi|$ term. Leaving it in until the Schwarz-inequality step yields

$$
\int(2 \sqrt{2} t|\omega|-s)^{2} d \mu \geq \frac{\left[\int\left|2 \nabla_{A} \Phi\right|^{2} d \mu+\int|\Phi|^{4} d \mu\right]^{2}}{\int|\Phi|^{4} d \mu} \geq \int|\Phi|^{4} d \mu+8 \int\left|\nabla_{A} \Phi\right|^{2} d \mu
$$


and one therefore concludes that

$$
t\left[4 \pi c_{1} \cdot[\omega]-\int_{M} s \frac{|\omega|}{\sqrt{2}} d \mu\right]+\frac{1}{8}\left[\int_{M} s^{2} d \mu-32 \pi^{2} c_{1}^{2}\right] \geq \int_{M}\left|\nabla_{A} \Phi\right|^{2} d \mu
$$

which we may rewrite as

$$
\left[4 \pi c_{1} \cdot[\omega]-\int_{M} s \frac{|\omega|}{\sqrt{2}} d \mu\right]+\frac{1}{8 t}\left[\int_{M} s^{2} d \mu-32 \pi^{2} c_{1}^{2}\right] \geq \int_{M}\left|\nabla_{A_{t}} \Psi_{t}\right|^{2} d \mu .
$$

It follows that

$$
\int_{M} s \frac{|\omega|}{\sqrt{2}} d \mu=4 \pi c_{1} \cdot[\omega] \Longrightarrow \lim _{t \rightarrow \infty}\left\|\nabla_{A_{t}} \Psi_{t}\right\|_{L^{2}}=0 .
$$

Since $\sigma\left(\Psi_{t}\right)$ is the contraction of a parallel field with $\Psi_{t} \otimes \bar{\Psi}_{t}+\bar{\Psi}_{t} \otimes \Psi_{t}$, and because we have a uniform $C^{0}$ bound for $\left|\Psi_{t}\right|$, application of the Leibniz rule yields

$$
\lim _{t \rightarrow \infty}\left\|\nabla \sigma\left(\Psi_{t}\right)\right\|_{L^{2}}=0
$$

whenever equality holds in Theorem 2 . Here the connection $\nabla$ on self-dual 2forms is $t$-independent, and is actually the one induced by the (torsion-free) Levi-Civita connection of $g$. Since $\omega$ is harmonic, it follows that

$$
\lim _{t \rightarrow \infty}\left\|\left(d^{+}\right)^{*}\left(\sigma\left(\Psi_{t}\right)-\omega\right)\right\|_{L^{2}}=\lim _{t \rightarrow \infty}\left\|\nabla \cdot \sigma\left(\Psi_{t}\right)\right\|_{L^{2}}=0,
$$

where $\left(d^{+}\right)^{*}=-\star d$ is the adjoint of $d^{+}: \Gamma \wedge^{1} \rightarrow \Gamma \wedge^{+}$with respect to $g$. Because the complex

$$
0 \rightarrow \Gamma \wedge^{0} \stackrel{d}{\longrightarrow} \Gamma \wedge^{1} \stackrel{d^{+}}{\longrightarrow} \Gamma \wedge^{+} \rightarrow 0
$$

is elliptic, the Gårding inequality

$$
\|\phi\|_{L_{1}^{2}} \leq C\left(\left\|\left(d^{+}\right)^{*} \phi\right\|_{L^{2}}+\|\phi\|_{L^{2}}\right) \forall \phi \in \Gamma \wedge^{+},
$$

applied to $\phi=\sigma\left(\Psi_{t}\right)-\omega$, then tells us that $\sigma\left(\Psi_{t}\right) \rightarrow \omega$ in the Sobolev space $L_{1}^{2}$. It follows that

$$
\|\nabla \omega\|_{L^{2}}=\lim _{t \rightarrow \infty}\left\|\nabla \sigma\left(\Psi_{t}\right)\right\|_{L^{2}}=0,
$$

and hence that $\nabla \omega=0$. Thus equality in Theorem 2 implies that $g$ is Kähler, as claimed.

By the same reasoning, we also get 
Theorem 3. Let $M^{4}$ be a smooth compact oriented 4-manifold with $b^{+}>1$ for which there is a spin ${ }^{c}$ structure $c$ such that the Seiberg-Witten invariant $n_{c}(M)$ is non-zero. Let $g$ be any Riemannian metric on $M$, and let $\omega$ be any $g$-self-dual harmonic 2-form. Then the scalar curvature $s$ of $g$ satisfies

$$
\int s \frac{|\omega|}{\sqrt{2}} d \mu \leq 4 \pi c_{1} \cdot[\omega]
$$

If $[\omega] \neq 0$, moreover, equality is achieved iff $g$ is Kähler with respect to some c-compatible complex structure $J$ and $\omega$ is a constant positive multiple of the Kähler form of $(M, g, J)$.

Recall [7] that an almost-Kähler manifold is a triple $(M, g, J)$, where $J$ is an almost complex structure on $M$ and $g$ is a $J$-invariant Riemannian metric, such that the 2-form $\omega(\cdot, \cdot)=g(J \cdot, \cdot)$ is closed. The symplectic form $\omega$ is then called the almost-Kähler form of $(M, g, J)$.

Corollary 1. Let $\left(M^{4}, g, J\right)$ be an almost-Kähler manifold. Then the scalar curvature $s$ of $g$ satisfies

$$
\int_{M} s d \mu \leq 4 \pi c_{1} \cdot[\omega]
$$

where $c_{1}$ is the first Chern class of $(T M, J)$ and $\omega$ is the almost-Kähler form. Moreover, equality is achieved iff $(M, g, J)$ is Kähler.

Proof. On any almost-Kähler manifold $\left(M^{4}, g, J\right)$, the almost-Kähler form is a harmonic self-dual form with $|\omega| \equiv \sqrt{2}$. Since Taubes [23] has shown that the perturbed Seiberg-Witten invariant of any symplectic 4-manifold is non-zero, the result therefore follows from the preceding theorems.

For related results proved by other methods, see [4].

\section{Genericity and Self-Duality.}

In light of Corollary 2, it is easy to see that Theorem 2 gives rise to an estimate of Yamabe invariants whenever the harmonic 2-form has empty zero locus. To generalize this, we will need to understand the manner in which harmonic forms vanish for generic metrics. The needed information is provided by the following result, the essence of which is contained in unpublished work of Taubes [22, Lemma B.3]. 
Proposition 1 (Taubes). Let $M$ be a smooth oriented 4-manifold with $b_{+} \geq 1$, and let $\mathcal{M}$ denote the space of $C^{k, \alpha}$ Riemannian metrics $g$ on $M$ for some $k \geq 2, \alpha \in(0,1)$. Choose a codimension- $\left(b_{+}-1\right)$ subspace $V \subset H^{2}(M, \mathbf{R})$ on which the intersection form has Lorentzian signature, and let $V_{1}$ be a connected component of the hyperboloid $\left\{[\omega] \in V \mid[\omega]^{2}=: 1\right\}$. For each $g \in \mathcal{M}$, let $\omega_{g}$ be the unique self-dual g-harmonic form with $\left[\omega_{g}\right] \in V_{1}$. Then

\section{$\left\{g \mid \omega_{g}\right.$ is transverse to the zero section of $\left.\wedge_{g}^{+}\right\} \subset \mathcal{M}$}

is a subset of the second Baire category.

Proof. Let $\Lambda^{+} \rightarrow M \times \mathcal{M}$ be the $C^{k}$ rank-3 vector bundle whose restriction to any slice $M \times\{g\}$ is the bundle $\wedge_{g}^{+} \rightarrow M$ of self-dual 2-forms determined by the metric $g$. Let $\Omega$ denote the section of $\Lambda^{+}$defined by

$$
\left.\Omega\right|_{M \times\{g\}}=\omega_{g} .
$$

By a straight-forward application of the Banach-space inverse function theorem and the interior Schauder estimates [6], the map $\mathcal{M} \ni g \rightarrow \omega_{g} \in$ $C^{k, \alpha}\left(\wedge^{2}\right)$ can be seen to be smooth, and this implies that $\Omega$ is a $C^{k}$ section of $\Lambda^{+}$.

Now $\Omega$ turns out to actually be transverse to the zero section of $\Lambda^{+}$. To see this, let $g \in \mathcal{M}$ and observe that for any other $\tilde{g} \in \mathcal{M}$, the bundle $\wedge_{\tilde{g}}^{+} \subset \wedge^{2}$ may be thought of as the graph of a homomorphism $h: \wedge_{g}^{+} \rightarrow \wedge_{g}^{-}$, and so in particular may be identified with $\wedge_{g}^{+}$via the projection $\wedge^{2} \rightarrow \wedge_{g}^{+}$. If $\tilde{g}$ is conformally rescaled so as to have the same volume form of $g$, moreover, and is we identify $\mathcal{H o m}\left(\wedge^{+}, \wedge^{-}\right)$with $\odot_{0}^{2} T^{*} M$ in the usual way, then one has $\tilde{g}=g+h+O\left(|h|^{2}\right)$. Using these identifications, one can then check that

$$
\left.\frac{d}{d t} \omega_{g+t h}\right|_{t=0}=-2 G\left[d^{+} d^{*} h(\omega)\right]
$$

by simply using only the facts that $\omega_{\tilde{g}}$ is closed and depends differentiably on $\tilde{g}$; here $G$ is the Green's operator of the Hodge Laplacian $d d^{*}+d^{*} d$ of $g$ on 2-forms. If $\omega$ vanishes at $p \in M$, it therefore suffices to show that $h \in \Gamma \mathcal{H o m}\left(\wedge^{+}, \wedge^{-}\right)=\Gamma \odot_{0}^{2} T^{*} M$ may be chosen so that $\left.G\left[d^{+} a^{* *} h(\omega)\right]\right|_{p}$ is any desired element $\phi$ of $\left.\Lambda_{g}^{+}\right|_{p}$. If $\hat{\phi}$ is the delta-function-like 2-current $\hat{\phi}(\psi)=\left\langle\left.\psi\right|_{p}, \phi\right\rangle_{g}$ corresponding to a non-zero $\left.\phi \in \wedge_{g}^{+}\right|_{p}$, however, this is equivalent to the assertion that the current $d^{-} d^{*} G \hat{\phi}$ is not identically zero on the open subset of $M$ where $\omega_{g} \neq 0$. Now the latter open subset is also 
dense in $M$ by unique continuation. Since $G \hat{\phi}$ is $C^{2}$ on $M-p$ by elliptic regularity, it therefore suffices to show that $d d^{*} G \hat{\phi}$ is not identically zero on $M-p$. But in geodesic normal coordinates $x^{j}$ about $p$, one has an expansion [5]

$$
G(\hat{\phi})=\frac{1}{4 \pi^{2}} \frac{\phi}{|x|^{2}}+O(\log |x|)
$$

where the Hessian of the $O(\log |x|)$ term is $O\left(1 /|x|^{2}\right)$; here $\phi$ has been extended as tensor field such that $\left.\nabla \phi\right|_{p}=0$. Assuming without loss of generality that $|\phi|=\sqrt{2}$ and rotating our coordinates as necessary, we may arrange, after transplanting the standard complex structure $J$ and complex codinates $\left(z^{1}, z^{2}\right)$ on on $\mathbf{R}^{4}=\mathbf{C}^{2}$ to our coordinate chart, that $\phi=g(J \cdot, \cdot)$, so that

$$
\begin{aligned}
d d^{*} G(\hat{\phi}) & =-\frac{1}{4 \pi^{2}} d J d\left(\frac{1}{r^{2}}\right)+O\left(\frac{1}{r^{2}}\right) \\
& =\frac{i\left(\left|z^{2}\right|^{2}-\left|z^{1}\right|^{2}\right)\left(d z^{1} \wedge d \bar{z}^{1}-d z^{2} \wedge d \bar{z}^{2}\right)+4 \Im m\left(\bar{z}^{1} z^{2} d z^{1} \wedge d \bar{z}^{2}\right)}{2 \pi^{2} r^{6}} \\
& +O\left(\frac{1}{r^{2}}\right)
\end{aligned}
$$

is indeed non-zero. Thus $\Omega$ is transverse to the zero section, as claimed.

Consequently, the zero locus $Z \subset M \times \mathcal{M}$ of $\Omega$ is a $C^{k}$ Banach submanifold of codimension 3, and the induced projection $\wp: Z \rightarrow \mathcal{M}_{[\omega]}$ is Fredholm, of index $4-3=1$. Since $\wp$ is a $C^{k} \operatorname{map}$, and $k>\max (\operatorname{index}(\wp), 0)$, the Smale-Sard Theorem [21] tells us that the set of regular values of $\wp$ is a set of the second Baire category. But this set of regular values is precisely the set of $C^{k, \alpha}$ metrics for which $\omega_{g}$ is transverse to the zero section.

This immediately implies the technical result we'll actually use:

Corollary 2. Let $g$ be a $C^{2}$ Riemannian metric on a compact oriented 4manifold $M$, and let $\omega \not \equiv 0$ be a self-dual harmonic form on $(M, g)$. Then for any integer $r \geq 2$ there is a sequence $\left\{g_{j}\right\}$ of $C^{r}$ metrics on $M$ and $a$ sequence $\left\{\omega_{j}\right\}$ of closed 2-forms such that

(a) $\lim _{j \rightarrow \infty} g_{j}=g$ in the $C^{2}$ topology;

(b) $\omega_{j}$ is self-dual with respect to $g_{j}$;

(c) $\omega_{j}$ is transverse to the zero section of $\wedge_{g_{j}}^{+} \rightarrow M$; and

(d) $\lim _{j \rightarrow \infty} \omega_{j}=\omega$ in the $C^{1}$ topology. 
Proof. Let $V \subset H^{2}(M, \mathbf{R})$ be spanned by $[\omega]$ and the cohomological image of the anti-self-dual $g$-harmonic forms; and let $V_{1}$ be the sheet of the unit hyperboloid in $V$ which contains $\lambda[\omega]$ for some $\lambda>0$. For each $C^{2}$ metric $\hat{g}$ we then have a unique self-dual harmonic 2 -form $\omega_{\hat{g}}$ with $\left[\omega_{\hat{g}}\right] \in V_{1}$. By Proposition 3, $\omega_{\hat{g}}$ is transverse to the zero section for a dense set of $C^{r, \alpha}$ metrics $\hat{g}$, and this set is automatically also dense in the space of $C^{2}$ metrics. Let $g_{j}$ be a sequence of such metrics converging to $g$ in the $C^{2}$ topology, and set $\omega_{j}=\omega_{g_{j}} / \lambda$. Now, for any $\alpha \in(0,1), \hat{g} \mapsto \omega_{\hat{g}}$ is a smooth map from $C^{1, \alpha}$ metrics to $C^{1, \alpha}$ forms, so $\omega_{j} \rightarrow \omega$ in the $C^{1, \alpha}$ topology, and hence in the $C^{1}$ topology, as claimed.

\section{Yamabe Invariants.}

Let $M$ be a smooth compact oriented 4-manifold, and let [ $g$ ] be a conformal class of metrics on $M$. The Yamabe constant of the conformal class is then defined by

$$
Y_{[g]}=\inf _{g \in[g]} \frac{\int_{M} s_{g} d \mu_{g}}{\sqrt{\int_{M} d \mu_{g}}} .
$$

Since Hodge star operator $\star: \wedge^{2} \rightarrow \wedge^{2}$ is conformally invariant, the corresponding eigenspace decomposition

$$
\wedge^{2}=\wedge^{+} \oplus \wedge^{-}
$$

of the 2-forms into self-dual and anti-self-dual parts is independent of a choice of $g \in[g]$.

Theorem 4. Let $(M,[g])$ be an oriented conformal Riemannian 4manifold, and let $\omega \not \equiv 0$ be a closed 2-form which is self-dual with respect to [g]. Suppose that $b^{+}(M)=1$ and that the perturbed Seiberg-Witten invariant $p_{c}\left(M, \mathcal{C}^{+}\right)$is non-zero for some spin ${ }^{c}$ structure $c$, where $\mathcal{C}^{+} \subset H^{2}(M, \mathbf{R})$ is the nappe containing $[\omega]$. Then the Yamabe constant of $[g]$ satisfies

$$
Y_{[g]} \leq \frac{4 \pi c_{1} \cdot[\omega]}{\sqrt{[\omega]^{2} / 2}}
$$

where $c_{1}$ is the first Chern class of $c$.

Proof. We may assume that the graph of the $[g]$-harmonic 2-form $\omega$ is transverse to the zero section of $\wedge^{+}$. Indeed, Corollary 3 tells us that the set of 
conformal classes with this property is dense, and both sides of the inequality are continuous functions with respect to the $C^{2}$-topology [3].

Let $\varphi: \mathbf{R} \rightarrow \mathbf{R}$ be a smooth function with

- $\varphi(x)=\frac{1}{2}$ when $x \leq \frac{1}{4}$;

- $\varphi(x)=x$ when $x \geq 1$; and

- $0 \leq \varphi^{\prime}(x) \leq 1$ for all $x$.

Let $g$ be any representative of $[g]$, and for each $\delta>0$ define a smooth positive function by $v=v_{\delta}=\delta \varphi\left(|\omega|_{g} / \delta\right) \leq|\omega|_{g}$. Let

$$
U_{\delta}=\left\{\left.p \in M|| \omega\right|_{g} \leq \delta\right\} .
$$

Notice that

- $|d v|_{g}<C$

- $\frac{\delta}{2} \leq v \leq \delta$ on $U_{\delta}$;

- $v \equiv \frac{\delta}{2}$ on $U_{\delta / 4}$; and

- $v \equiv|\omega|_{g}$ on $M-U_{\delta}$,

where $C$ is some constant independent of $\delta$. Moreover, there is a number $\delta_{0} \in(0,1)$ such that for all $\delta \in\left(0, \delta_{0}\right)$

- $U_{\delta}$ is a smooth manifold with boundary; and

- $\operatorname{Vol}_{g}\left(U_{\delta}\right)<C \delta^{3}$.

Notice that we are now exploiting the hypothesis that $[g]$ is generic.

For any $\delta \in\left(0, \delta_{0}\right)$, consider the smooth conformal metric $\hat{g}=v g \in[g]$. One has

$$
|\omega|_{\hat{g}}=v^{-1}|\omega|_{g} \leq 1
$$

and $|\omega|_{\hat{g}} \equiv 1$ on $M-U_{\delta}$. Moreover, the continuous function

$$
\psi=v^{-1}|\omega|_{g}=|\omega|_{\hat{g}}: M \rightarrow[0,1]
$$

is smooth on $M-U_{\delta / 4}$, with $|d \psi|_{g} \leq 4 C \delta^{-1}$ on this set, so

$$
\begin{aligned}
\left|\int_{M} s_{\hat{g}} d \mu_{\hat{g}}-\int_{M} s_{\hat{g}}\right| \omega\left|\hat{g} d \mu_{\hat{g}}\right| & =\left|\int_{U_{\delta}} s_{\hat{g}}(1-\psi) d \mu_{\hat{g}}\right| \\
& =\left|\int_{U_{\delta}}(1-\psi)\left[3 \Delta v+s_{g} v+\frac{3}{2} \frac{|d v|_{g}^{2}}{v}\right] d \mu_{g}\right|
\end{aligned}
$$




$$
\begin{aligned}
\leq & 3\left|\int_{U_{\delta}}(1-\psi) \Delta v d \mu_{g}\right| \\
& +\left|\int_{U_{\delta}}(1-\psi) s_{g} v d \mu_{g}\right|+\frac{3}{2}\left|\int_{U_{\delta}}(1-\psi) \frac{|d v|_{g}^{2}}{v} d \mu_{g}\right| \\
\leq & 3\left|\int_{U_{\delta}-U_{\delta / 4}}(1-\psi) \Delta v d \mu_{g}\right| \\
& +\int_{U_{\delta}}\left|s_{g}\right| v d \mu_{g}+\frac{3}{2} \int_{U_{\delta}} \frac{|d v|_{g}^{2}}{v} d \mu_{g} \\
\leq & 3\left|\int_{\partial U_{\delta} \cup \partial \bar{U}_{\delta / 4}}(1-\psi) \star d v+\int_{U_{\delta}-U_{\delta / 4}}\langle d \psi, d v\rangle_{g} d \mu_{g}\right| \\
& +\left(\max \left|s_{g}\right|\right) C \delta^{4}+C^{2} \frac{3}{\delta} C \delta^{3} \\
\leq & 3 C \frac{4 C}{\delta} C \delta^{3}+C\left(\max \left|s_{g}\right|\right) \delta^{4}+3 C \delta^{2} \leq \hat{C} \delta^{2}
\end{aligned}
$$

because $\psi \equiv 1$ on $\partial U_{\delta}$ and $d v \equiv 0$ on $\partial U_{\delta / 4}$. On the other hand,

$$
\int_{M} d \mu_{\hat{g}} \geq \int_{M}|\omega|_{\hat{g}}^{2} d \mu_{\hat{g}}=\int_{M} \omega \wedge \omega=[\omega]^{2} .
$$

So given $\epsilon>0$, consider the metric $\hat{g}$ corresponding to some $\delta$ with $\hat{C} \delta^{2}<$ $\epsilon \sqrt{[\omega]^{2}}$. By Theorem 2, this metric satisfies

$$
\frac{\int_{M} s_{\hat{g}} d \mu_{\hat{g}}}{\sqrt{\int_{M} d \mu_{\hat{g}}}}<\frac{\int_{M} s_{\hat{g}}|\omega| \hat{g} d \mu_{\hat{g}}}{\sqrt{[\omega]^{2}}}+\epsilon \leq \frac{4 \sqrt{2} \pi c_{1} \cdot[\omega]}{\sqrt{[\omega]^{2}}}+\epsilon .
$$

Hence

$$
Y_{[g]}=\inf _{\hat{g} \in[g]} \frac{\int_{M} s_{\hat{g}} d \mu_{\hat{g}}}{\sqrt{\int_{M} d \mu_{\hat{g}}}} \leq \frac{4 \pi c_{1} \cdot[\omega]}{\sqrt{[\omega]^{2} / 2}}
$$

as claimed.

Theorem 5. Equality is achieved in Theorem 4 iff there is a Yamabe minimizer $g \in[g]$ which is Kähler, with Kähler form $\omega$.

Proof. We may assume that $c_{1} \cdot[\omega]>0$, since otherwise the result follows from the theory of the unperturbed Seiberg-Witten equations [12]. 
Let us begin by showing that equality can only be achieved if the harmonic form $\omega$ is nowhere zero. Since $Y_{[g]}>0$, we may therefore choose a metric $g$ of positive scalar curvature in $[g]$. By Corollary 3, we can find be a sequence $g_{j}$ of positive-scalar-curvature metrics converging to $g$ in the $C^{2}$ topology such that the corresponding harmonic forms $\omega_{j}$, normalized so that $\left[\omega_{j}\right]^{2}=[\omega]^{2}$, are transverse to the zero section and converge to $\omega$ in the $C^{1}$ topology. For each $j$ there is an $\epsilon_{j}>0$ such that

$$
\frac{\int\left[s_{g} u^{2}+6|d u|_{g}^{2}\right] d \mu_{g}}{\sqrt{\int u^{4} d \mu_{g}}} \leq\left(1+\epsilon_{j}\right) \frac{\int\left[s_{g_{j}} u^{2}+6|d u|_{g_{j}}^{2}\right] d \mu_{g_{j}}}{\sqrt{\int u^{4} d \mu_{g_{j}}}}
$$

for every $u \in L_{1}^{2}$, and moreover one can take $\epsilon_{j} \rightarrow 0$ as $j \rightarrow \infty$. Now let $u_{j}=\left[\delta_{j} \varphi\left(\left|\omega_{j}\right|_{g_{j}} / \delta_{j}\right)\right]^{1 / 2}$, where $\varphi$ is as in the proof of Theorem 4 and $\delta_{j}$ is chosen so small that

$$
\frac{\int\left[s_{g_{j}} u^{2}+6|d u|_{g_{j}}^{2}\right] d \mu_{g_{j}}}{\sqrt{\int u^{4} d \mu_{g_{j}}}} \leq \frac{4 \pi c_{1} \cdot\left[\omega_{j}\right]}{\sqrt{[\omega]^{2} / 2}}+\epsilon_{j},
$$

for each $j$, and is also chosen in such a manner that $\lim _{j \rightarrow \infty} \delta_{j}=0$. Since $\left[\omega_{j}\right] \rightarrow[\omega]$, it follows that

$$
\frac{\int\left[s_{g} u_{j}^{2}+6\left|d u_{j}\right|_{g}^{2}\right] d \mu_{g}}{\sqrt{\int u_{j}^{4} d \mu_{g}}} \longrightarrow \frac{4 \pi c_{1} \cdot\left[\omega_{j}\right]}{\sqrt{[\omega]^{2} / 2}}=Y_{[g]}
$$

and the $u_{j}$ are in particular bounded in $L_{1}^{2}$ because $\int u_{j}^{4} d \mu \rightarrow[\omega]^{2}$. By. passing to a subsequence, we may therefore assume that the $u_{j}$ are weakly convergent to some $u \in L_{1}^{2}$. But this implies that $u$ is the unique weak limit of the $u_{j}$ in $L^{2}$. However, by construction, $u_{j} \rightarrow \sqrt{|\omega|_{g}}$ in $C^{0}$, and hence in $L^{2}$. We therefore have $u=\sqrt{|\omega|_{g}}$, so that $\sqrt{|\omega|_{g}} \in L_{1}^{2}$. Now since $u$ is the weak limit of the $u_{j}$,

$$
\|u\|_{L_{1}^{2}} \leq \lim _{j \rightarrow \infty}\left\|u_{j}\right\|_{L_{1}^{2}}
$$

whereas we also know that

$$
\|u\|_{L^{4}}^{4}=\|\sqrt{|\omega|}\|_{L^{4}}^{4}=[\omega]^{2}=\lim _{j \rightarrow \infty}\left\|u_{j}\right\|_{L^{4}}^{4}
$$

by construction. Hence

$$
\frac{\int\left[s_{g} u^{2}+6|d u|_{g}^{2}\right] d \mu_{g}}{\sqrt{\int u^{4} d \mu_{g}}} \leq \lim _{j \rightarrow \infty} \frac{\int\left[s_{g} u_{j}^{2}+6\left|d u_{j}\right|_{g}^{2}\right] d \mu_{g}}{\sqrt{\int u_{j}^{4} d \mu_{g}}}=Y_{[g]}
$$




$$
=\inf _{\hat{u} \in L_{1}^{2}-0} \frac{\int\left[s_{g} \hat{u}^{2}+6|d \hat{u}|_{g}^{2}\right] d \mu_{g}}{\sqrt{\int \hat{u}^{4} d \mu_{g}}}
$$

so that $u \in L_{1}^{2}$ is a minimizer of the Yamabe functional, and is automatically a weak solution of the associated Euler-Lagrange equation

$$
6 \Delta u+s u=s_{0} u^{3},
$$

where $s_{0}=4 \sqrt{2} \pi \frac{c_{1} \cdot[\omega]}{[\omega]^{2}}$. Since $u=\sqrt{|\omega|} \in C^{0,1 / 2}$, elliptic regularity [1], applied to (4.1), implies that $u \in C^{2,1 / 2}$, and an application [1, Prop. 3.75] of the maximum principle to (4.1) therefore shows that $u \geq 0$ must either be strictly positive or vanish identically. Hence $|\omega|=u^{2} \neq 0$, and $\omega \neq 0$, as claimed.

We may therefore define an almost-Kähler metric $\hat{g} \in[g]$ by $\hat{g}=\sqrt{2}|\omega|_{g} g$. Since $\int d \mu_{\hat{g}}=[\omega]^{2} / 2$, Corollary 2 tells us that

$$
\frac{\int s_{\hat{g}} d \mu_{\hat{g}}}{\sqrt{\int d \mu_{\hat{g}}}} \leq \frac{4 \pi c_{1} \cdot[\omega]}{\sqrt{[\omega]^{2} / 2}}
$$

with equality iff $g$ is Kähler. Since the right-hand side is $Y_{[g]}$ by hypothesis, however, we also have a tautological inequality in the other direction, and the two sides must be equal. Thus $\hat{g}$ is both a Yamabe minimizer and a Kähler metric, as desired.

Now recall that the Yamabe invariant (sometimes called the sigma constant) of a smooth $n$-manifold $M$ is defined to be

$$
Y(M)=\sup _{[g]} \inf _{g \in[g]} \frac{\int_{M} s_{g} d \mu_{g}}{\left(\int_{M} d \mu_{g}\right)^{\frac{n-2}{n}}}=\sup _{[g]} Y_{[g]}
$$

where $[g]$ is allowed to vary over the space of conformal class of: smooth Riemannian metrics on $M$.

Theorem 6 (Main Theorem). The Yamabe invariant of the complex projective plane is given by

$$
Y\left(\mathbf{C P}_{2}\right)=12 \sqrt{2} \pi .
$$

Moreover, a conformal class $[g]$ on $\mathbf{C P}_{2}$ satisfies $Y_{[g]}=Y\left(\mathbf{C P}_{2}\right)$ iff there is a diffeomorphism $\Phi: \mathbf{C P}_{2} \rightarrow \mathbf{C P}_{2}$ such that $\Phi^{*}[g]$ is the conformal class of the Fubini-Study metric. 
Proof. Let $c$ be the spin ${ }^{c}$ structure induced by the usual complex structure on $\mathbf{C P}_{2}$, and let $\mathcal{C}^{+}$denote the nappe containing $c_{1}$. Then the perturbed Seiberg-Witten invariant $p_{c}\left(\mathbf{C P}_{2}, \mathcal{C}^{+}\right) \neq 0$, as may be seen in a variety of ways $[10,11,15,23]$. Now since $b_{-}=0$, the class $[\omega]=c_{1}$ is self-dual with respect to any conformal class $[g]$, and we therefore always have

$$
Y_{[g]} \leq 4 \pi \frac{c_{1} \cdot c_{1}}{\sqrt{c_{1}^{2} / 2}}=12 \sqrt{2} \pi
$$

with equality iff $[\mathrm{g}]$ is represented by a Kähler metric metric of constant positive scalar curvature. For such a metric, however, the Ricci form is harmonic and since $b_{-}=0$, this implies that the Ricci form is a constant multiple of the Kähler form, and the metric is therefore Kähler-Einstein, with positive scalar curvature. But it follows, for example, from the Enriques-Kodaira classification [2] and Matsushima's theorem on isometry and automorphism groups [17] that any such metric on the smooth 4-manifold $\mathbf{C P}_{2}$ is the standard Fubini-Study metric up to diffeomorphisms and rescaling.

Theorem 7. Let $M$ be a smooth compact orientable manifold with $b_{1}=0$ and $b_{2}=1$. Then

$$
Y(M) \leq 12 \sqrt{2} \pi
$$

Moreover, $M$ admits a conformal class with $Y_{[g]}=12 \sqrt{2} \pi$ iff $M$ is diffeomorphic to $\mathbf{C P}_{2}$.

Proof. If $M$ does not admit metrics of positive scalar curvature, there is nothing to prove. Otherwise, let $c$ be a $\operatorname{spin}^{c}$-structure with $c_{1}^{2}=2 \chi+3 \tau=9$, and let $\mathcal{C}^{+}$be the nappe containing $[\omega]=c_{1}$. Since there is a metric of positive scalar curvature on $M$, there are no solutions of the unperturbed Seiberg-Witten equations for any metric on $M$, and because $c_{1} \cdot[\omega]>0$, the wall-crossing formula $[10,15]$ therefore allows us to conclude that the perturbed invariant $p_{c}\left(M, \mathcal{C}^{+}\right)$is \pm 1 . As in the last proof, we therefore have

$$
Y_{[g]} \leq 4 \pi \frac{c_{1} \cdot c_{1}}{\sqrt{c_{1}^{2} / 2}}=12 \sqrt{2} \pi
$$

for every conformal class, with equality only if [g] is represented by a KählerEinstein metric of positive scalar curvature. The diffeomorphism statement therefore follows from the classification [2] of complex surfaces with $c_{1}>$ 0 .

Exactly the same reasoning also proves the following: 
Proposition 2. Let $(M, J)$ be a compact complex surface with $c_{1}>0$, and let $[g]$ be any conformal class on $M$ for which $c_{1}$ is self-dual. Then

$$
Y_{[g]} \leq 4 \pi \sqrt{2 c_{1}^{2}(M)}
$$

with equality iff $[g]$ can be represented by a Kähler-Einstein metric adapted to some deformation of $J$.

However, this will not help to to compute the Yamabe invariant of manifolds other than $\mathbf{C P}_{2}$. For example, combining our results with the connect-sum estimate of $\mathrm{O}$. Kobayashi [9] tells one that $Y\left(\mathbf{C P}_{2} \# k \overline{\mathbf{C P}}_{2}\right) \geq$ $Y\left(\mathbf{C P}_{2}\right)=12 \sqrt{2} \pi>4 \pi \sqrt{2 c_{1}^{2}\left(\mathbf{C P}_{2} \# k \overline{\mathbf{C P}}_{2}\right)}$ for any $k>0$. This of course does not contradict the above Proposition, since $c_{1}$ is not self-dual for most conformal classes as soon as $b_{2}>1$. A reasonable conjecture would seem to be that that $Y\left(\mathbf{C P}_{2} \# k \overline{\mathbf{C P}}_{2}\right)=Y\left(\mathbf{C P}_{2}\right)=12 \sqrt{2} \pi$ for all $k$.

Let us now conclude with a new, non-twistor-theoretic proof of a theorem of Poon [18]. Recall that an oriented conformal Riemannian manifold $(M,[g])$ is called self-dual if its Weyl curvature satisfies $W_{-}=0$. For example, the conformal class of the Fubini-Study metric on $\mathbf{C P}_{2}$ is self-dual.

Corollary 3 (Poon). Let $(M,[g])$ be a self-dual 4-manifold with $b_{1}=0$ and $b_{2}=1$. Suppose, moreover, that $Y_{[g]} \geq 0$. Then $(M,[g])$ is conformally isometric to $\mathbf{C P}_{2}$ equipped with the Fubini-Study metric.

Proof. Let $g \in[g]$ be a Yamabe minimizer. Since

$$
b_{+}-b_{-}=\tau=\frac{1}{12 \pi^{2}} \int_{M}\left[\left|W_{+}\right|^{2}-\left|W_{-}\right|^{2}\right] d \mu \geq 0,
$$

and $b_{+}+b_{-}=b_{2}=1$, we must have $b_{+}=1$ and $b_{-}=0$. Thus

$$
3=2 \chi-3 \tau=\frac{1}{4 \pi^{2}} \int_{M}\left[2\left|W_{-}\right|^{2}+\frac{s^{2}}{24}-\frac{|\stackrel{\circ}{r}|^{2}}{2}\right] d \mu \leq \frac{1}{96 \pi^{2}} \int_{M} s^{2} d \mu
$$

where $\stackrel{\circ}{r}$ denotes the trace-free Ricci tensor and $W_{-}=0$. Because $g$ was chosen to be a Yamabe minimizer, and so has constant scalar curvature, Theorem 4 tells us that

$$
\int_{M} s^{2} d \mu=\left(Y_{[g]}\right)^{2} \leq(12 \sqrt{2} \pi)^{2}=3\left(96 \pi^{2}\right),
$$


so the above inequality is actually an equality, forcing

$$
Y_{[g]}=12 \sqrt{2} \pi,
$$

and so implying that $M$ is diffeomeorphic to $\mathbf{C P}_{2}$. Moreover, Theorem 4 tells us that the diffeomeorphism can be chosen so that $[g]$ is the pull-back of the Fubini-Study conformal class.

Acknowledgements. The author would particularly like to thank Cliff Taubes for his help in connection with Proposition 3. He would also like to thank Mike Anderson and Matt Gursky for their helpful remarks on minimizing sequences in the Yamabe problem.

\section{References.}

[1] T. Aubin, Nonlinear analysis on Manifolds. Mong-Ampère Equations, Springer-Verlag, 1982.

[2] W. Barth, C. Peters and A. Van de Ven, Compact Complex Surfaces, Springer-Verlag, 1984.

[3] L. Bérard-Bergery, Scalar Curvature and Isometry Groups, in Spectra of Riemannian Manifolds, ed. Berger, Murakami, Ochiai, Kagai, Tokyo, 1983, pp. 9-28.

[4] D.E. Blair and S. Ianus, Critical Associated Metrics on Symplectic Manifolds, Non-Linear Problems in Geometry, Contemp. Math. 51 (1986) 23-29.

[5] E. Combet, Paramétrix et Invariants sur les Variétés Compactes, Ann. Sci. ENS 4 (1970) 247-271.

[6] A. Douglis and L. Nirenberg, Interior Estimates for Elliptic Systems of Partial Differential Equations, Comm. Pure Appl. Math. 8 (1955) 503-538.

[7] S.I. Goldberg, Integrability of Almost Kähler Manifolds, Proc. AMS 21 (1969) 96-100.

[8] M. Gromov and H.B. Lawson, Spin and Scalar Curvature in the Presence of the Fundamental Group, Ann. Math. 111 (1980) 209-230. 
[9] O. Kobayashi, Scalar Curvature of a Metric of Unit Volume, Math. Ann. 279 (1987) 253-265.

[10] P. Kronheimer and T. Mrowka, The Genus of Embedded Surfaces in the Complex Projective Plane, Math. Res. Lett. 1 (1994) 797-808.

[11] C. LeBrun, On the Scalar Curvature of Complex Surfaces, Geom. Func. An. 5 (1995) 619-628.

[12] C. LeBrun, Polarized 4-Manifolds, Extremal Kähler Metrics, and Seiberg-Witten Theory, Math. Res. Lett. 2 (1995) 653-662.

[13] C. LeBrun, Four-Manifolds without Einstein Metrics, Math. Res. Lett. 3 (1996) 133-147.

[14] J. Lee and T. Parker, The Yamabe Problem, Bull. Am. Math. Soc. 17 (1987) 37-91.

[15] T.J. Li and A. Liu, General Wall Crossing Formula, Math. Res. Lett. 2 (1995) 797-810.

[16] A. Lichnerowicz, Spineurs Harmoniques, C. R. Acad, Sci. Paris 257 (1963) 7-9.

[17] Y. Matsushima, Sur la Structure du Groupe d'Homéomeorphismes Analytiques d'une Certaine Variété Kählerienne, Nagoya Math. J. 11 (1957) 145-150.

[18] Y-S. Poon, Compact Self-Dual Manifolds with Positive Scalar Curvature, J. Diff. Geom. 24 (1986) 97-132.

[19] R. Schoen, Conformal Deformation of a Riemannian Metric to Constant Scalar Curvature, J. Diff. Geom. 20 (1984) 478-495.

[20] R. Schoen and S.-T. Yau, On the Structure of Manifolds with Positive Scalar Curvature, Man. Math. 28 (1979) 159-183.

[21] S. Smale, An Infinite Dimensional Version of Sard's Theorem Am. J. Math. 87 (1965) 861-866.

[22] C. H. Taubes, Self-Dual Connections on 4-Manifolds with Indefinite Intersection Matrix, Harvard preprint, 1982; published minus the relevant lemma in J. Diff. Geom. 19 (1984) 517-560. 
[23] C. H. Taubes, The Seiberg-Witten and Symplectic Forms, Math. Res. Lett. 1 (1995) 809-822.

[24] C. H. Taubes, The Seiberg-Witten and Gromov Invariants, Math. Res. Lett. 2 (1995) 221-238.

[25] E. Witten, Monopoles and Four-Manifolds, Math. Res. Lett. 1 (1994) 769-796.

Received May 30, 1996.

DePARTMENT OF MATHEMATICS

STATE UNIVERSiTY OF NEW YORK

STONY BROOK, NY 11794-3651

E-MAIL: CLAUDE@MATH.SUNYSB.EDU 